\title{
SOURCE TERM MODEL FOR VORTEX GENERATOR VANES IN A NAVIER-STOKES COMPUTER CODE
}

\author{
Kenrick A. Waithe $\dagger$ \\ NASA Langley Research Center \\ Hampton, VA
}

\begin{abstract}
A source term model for an array of vortex generators was implemented into a non-proprietary Navier-Stokes computer code, OVERFLOW. The source term models the side force created by a vortex generator vane. The model is obtained by introducing a side force to the momentum and energy equations that can adjust its strength automatically based on the local flow.

The model was tested and calibrated by comparing data from numerical simulations and experiments of a single low profile vortex generator vane on a flat plate. In addition, the model was compared to experimental data of an S-duct with 22 co-rotating, low profile vortex generators.

The source term model allowed a grid reduction of about seventy percent when compared with the numerical simulations performed on a fully gridded vortex generator on a flat plate without adversely affecting the development and capture of the vortex created. The source term model was able to predict the shape and size of the stream-wise vorticity and velocity contours very well when compared with both numerical simulations and experimental data. The peak vorticity and its location were also predicted very well when compared to numerical simulations and experimental data. The circulation predicted by the source term model matches the prediction of the numerical simulation. The source term model predicted the engine fan face distortion and total pressure recovery of the S-duct with 22 co-rotating vortex generators very well.

The source term model allows a researcher to quickly investigate different locations of individual or a row of vortex generators. The researcher is able to conduct a preliminary investigation with minimal grid generation and computational time.
\end{abstract}

\footnotetext{
†Aerospace Engineer, Configuration Aerodynamics Branch, Aerodynamics, Aerothermodynamics, and Acoustics Competency. Member AIAA.

This material is declared a work of the U.S. Govemment and is not subject to copyright protection in the United States
}

\section{Introduction}

Low profile vortex generating (VG) devices have shown to be useful for improving turbofan engineface distortion in the design of compact aircraft inlets [1]. Computational fluid dynamics (CFD) is a tool that can be used to predict the fan face distortion and in conjunction with experiments can be used to design new inlets. Before CFD can be used, researchers have to be sure that CFD analysis can model the complex flows associated with the vortices created by a VG. Allan et al. [2] have shown a comparison between a CFD analysis and experimental data of a single low profile vortex generator on a flat plate. Their results indicate that CFD analysis can accurately predict various aspects of the resulting vortex including size, shape, location, and decay.

The work conducted by Allan et al. was significant, but detailed modeling of multiple VG devices is impractical. A simplified model must be used. The small size of VGs requires very fine grids to model the VG and the region immediately surrounding the VG. As a result, the number of grids required to model the entire flow increases and with an increase in grids comes an increase in computational time. As the number of VGs is increased from one to more than twenty, the grid generation and computational time becomes enormous.

Bender et al. developed a source term model for modeling an array of VGs without generating grids for the VGs [3]. The model is obtained by introducing a side force to the momentum and energy equations that can adjust its strength automatically based on the local flow. The user grids the rest of the flow replacing the VG with a source term that models the side force created by the VG or an array of VGs.

This paper describes the implementation of this source term model for an array of VGs in the Reynolds Averaged Navier-Stokes (RANS) CFD computer code OVERFLOW version 1.8s [4] based on the method described by Bender et al. [3]. In addition, this report presents the results of CFD analysis utilizing the source term model on a single, low profile VG on a flat plate. The results are compared to experimental results and to a CFD 
analysis where a grid was generated for the same low profile VG on a flat plate [2]. Also, CFD analysis using the source term model on an S-duct, the M2129 inlet, [5] to model 22 co-rotating VGs is compared to experimental results of the same inlet. The M2129 inlet is an S-duct with highly separated flows, and the application of VGs helps to keep the flow attached, thus reducing engine fan face distortion and improving fan face pressure recovery. Lastly, some preliminary CFD analysis on a flush mounted, boundary layer ingestion (BLI) inlet [6] will be presented. The BLI inlet results are presented to demonstrate the use of the model in preliminary design. In particular, the use of the model can aid in the placement of VGs in an inlet for the purposes of reducing fan face distortion and improving fan face pressure recovery.

\section{Nomenclature}

$\begin{array}{ll}\text { A } & \text { cross-sectional area } \\ \hat{b} & \text { unit vector parallel to VG, normal to surface } \\ \mathrm{b}_{1} & \text { component } 1 \text { of vector } \hat{b} \\ \mathrm{~b}_{2} & \text { component } 2 \text { of vector } \hat{b} \\ \mathrm{~b}_{3} & \text { component } 3 \text { of vector } \hat{b} \\ \mathrm{c} & \text { calibration constant } \\ \mathrm{dA} & \text { differential cross-sectional area } \\ \mathrm{DC} & \text { circumferential distortion descriptor } \\ \mathrm{K} & \text { cross-stream kinetic energy } \\ \mathrm{L} & \text { Length of BLI inlet } \\ \bar{L} & \text { side force } \\ \mathrm{M} & \text { Mach number } \\ \hat{n} & \text { unit vector normal to VG } \\ \mathrm{n}_{1} & \text { component } 1 \text { of vector } \hat{n} \\ \mathrm{n}_{2} & \text { component } 2 \text { of vector } \hat{n} \\ \mathrm{n}_{3} & \text { component } 3 \text { of vector } \hat{n} \\ \mathrm{p}_{0} & \text { total pressure in inlet } \\ \mathrm{p}_{0, \infty} & \text { free stream total pressure } \\ \mathrm{p}_{0} / \mathrm{p}_{0, \infty} & \text { total pressure ratio } \\ \mathrm{R}_{\mathrm{fan}} & \text { radius of inlet engine fan face } \\ \mathrm{R}_{\mathrm{t}} & \text { radius of inlet throat } \\ \mathrm{S}_{\mathrm{vg}} & \text { plan form surface area } \\ \hat{t} & \text { unit vector tangential to VG, normal to } \hat{b} \\ \mathrm{t}_{1} & \text { component } 1 \text { of vector } \hat{t} \\ \mathrm{t}_{2} & \text { component } 2 \text { of vector } \hat{t} \\ \mathrm{t}_{3} & \text { component } 3 \text { of vector } \hat{t} \\ \mathrm{u} & \text { component } 1 \text { of } \bar{U} \\ \mathrm{U}_{\infty} & \text { free stream velocity } \\ \bar{U} & \text { local velocity vector } \\ \mathrm{v} & \text { component } 2 \text { of } \bar{U} \\ \mathrm{~V}_{\mathrm{i}} & \text { cell volume } \\ \mathrm{V}_{\mathrm{M}} & \text { total volume of cells } \\ & \end{array}$

\begin{tabular}{|c|c|}
\hline w & component 3 of $\bar{U}$ \\
\hline $\mathbf{X}$ & axial direction \\
\hline $\mathrm{y}$ & cross stream direction \\
\hline $\mathbf{z}$ & normal direction \\
\hline$\alpha$ & angle of attack of VG to local flow \\
\hline$\kappa-\varepsilon$ & two-equation turbulence model \\
\hline$\kappa-\omega$ & two-equation turbulence model \\
\hline$\delta$ & boundary layer thickness \\
\hline$\frac{\Delta V_{i}}{V_{M}}$ & ratio of cell volume to total volume of cells \\
\hline$\Gamma$ & circulation \\
\hline$\rho$ & density \\
\hline$\omega$ & stream-wise vorticity \\
\hline$\omega_{\max }$ & maximum stream-wise vorticity \\
\hline$\partial \mathbf{v}$ & differential of $v$ \\
\hline$\partial w$ & differential of $w$ \\
\hline$\partial \mathrm{y}$ & differential of $y$ \\
\hline$\partial \mathbf{z}$ & differential of $\mathbf{z}$ \\
\hline
\end{tabular}

\section{Numerical Method}

The source term model, which was developed by Bender et al. [3], describes vortex generator modeling for Navier-Stokes codes. The basic methodology of the model is to introduce a side force, $\bar{L}_{i}$, that is normal to the local flow and is parallel to the surface. This side force is representative of the side force created by the VG being modeled. The final formulation for the side force is given by equation 1 .

$$
\vec{L}_{i}=c S_{v g} \frac{\Delta V_{i}}{V_{M}} \rho(\bar{U} \cdot \hat{n})(\bar{U} \times \hat{b})\left(\frac{\bar{U}}{|\bar{U}|} \cdot \hat{t}\right)
$$

In equation $1, \mathrm{c}$ is a calibration constant discussed later, $S_{\mathrm{vg}}$ is the planform area of the VG to be modeled, $\bar{U}$ is the local velocity vector, $\left(\Delta \mathrm{V}_{\mathrm{i}}\right) / \mathrm{V}_{\mathrm{M}}$ is the ratio of the cell volume to total volume of cells the source term is applied to, $\rho$ is the density of the fluid, and $\hat{b}, \hat{n}$, and $\hat{t}$ are unit vectors that specify the orientation of the VG. Figure 1 shows a schematic of the orientation of these vectors given 
the angle of attack of the VG to the local flow, $\alpha$. The computed side force is added to the discretized momentum and energy equations.

The source term model was implemented into the CFD code OVERFLOW version 1.8s. OVERFLOW solves the steady, compressible, Reynolds-Averaged Navier-Stokes (RANS) equations using the diagonal scheme of Pulliam and Chaussee [7]. The RANS equations are solved on structured grids using the overset grid framework of Steger et al. [8], which allows for complex geometries.

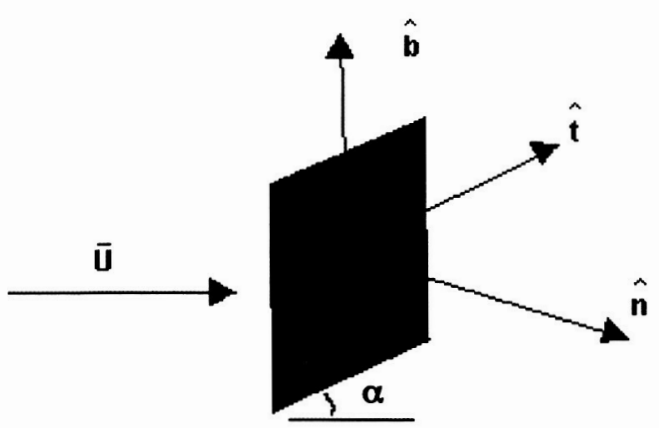

Figure 1. Schematic of vector orientation.

The source term model is invoked in OVERFLOW by specifying a region as a boundary condition to a small group of cells containing the VG to be modeled.

Top View

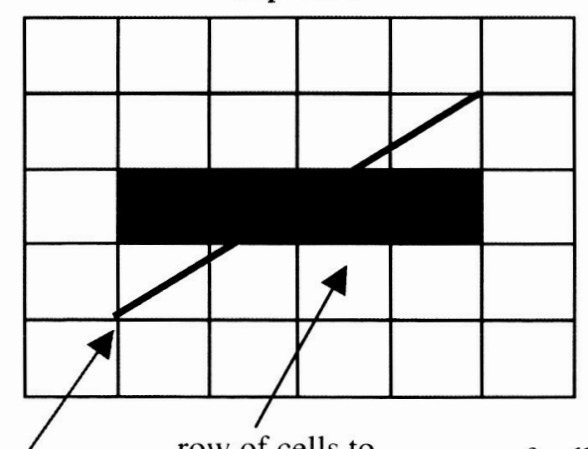

row of cells to span VG chord

row of cells to

VG being modeled span VG height

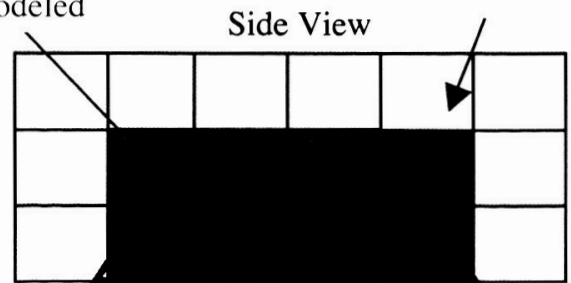

Figure 2. Schematic of cells selected for application of source term model.
In particular, a row or several rows of cells that span the chord and the height of the VG are selected as shown in figure 2 . The user also specifies the planform area, $S_{v g}$, and the angle of attack between the local flow and the VG, $\alpha$. The reader should note that the specific shape of the VG is not modeled by the rectangular selection of cells shown in figure 2 . The reason for the rectangular selection is because Allan et al. [2] demonstrated that the shape of low profile VGs is not important in determining the effects of the VG, but the surface area, $S_{v g}$, and height are.

\section{Flat Plate Study}

The source term model was tested by running simulations on a flat plate and implementing a source term to model a low profile VG. The grid for the flat plate used in this study is shown in figure 3 and comprises a large, coarse block grid for the flat plate and a smaller, finer block grid for capturing the vortex for a total of $1,888,000$ grid cells.

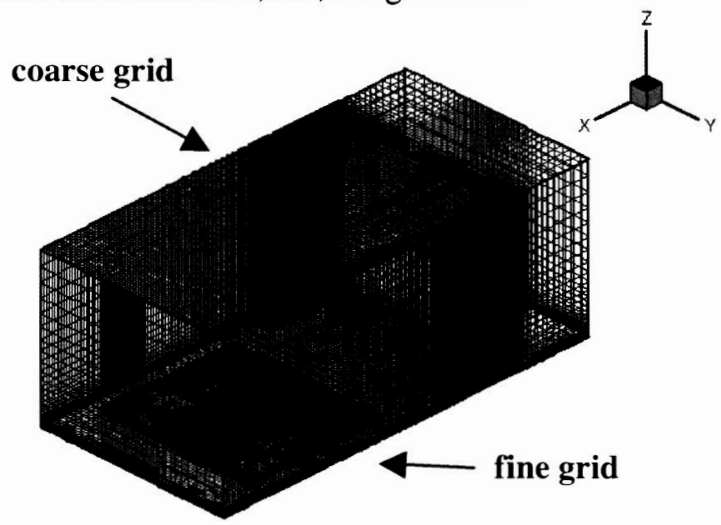

Figure 3. Computational domain for flat plate study.

A schematic of the VG being modeled is shown in figure 4 , which has a length to height ratio of approximately 7 , a planform area of $0.5772 \mathrm{~mm}^{2}$, and a $10^{\circ}$ angle of attack relative to the local flow.

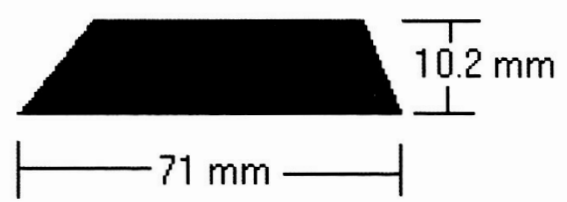

Figure 4. Schematic of VG being modeled.

All simulations were run at the test condition, which was a free-stream velocity, $U_{\infty}$, of $34 \mathrm{~m} / \mathrm{s}(M \approx 0.1)$. A total of 15 cases were run to explore the effects of varying the calibration constant, $c$, and the number of 
rows of cells used to span the chord of the VG being modeled. In particular, the calibration constant was arbitrarily varied from $c=0.5,1,2,5$, and 10 and the number of rows of cells varied from 1,3 , and a maximum of 11 , which was the number of rows of cells totally enclosing the VG.

The low-Mach preconditioning and multi-grid acceleration options of OVERFLOW were used to improve the convergence of the steady-state solution. All simulations were run using the two-equation ( $\kappa$ $\omega)$ Shear-Stress Transport (SST) turbulence model of Menter [9]. The SST model was used because the study conducted by Allan et al. [2] indicated the SST model does a better job than the Spalart and Allmaras (SA) turbulence model [10] predicting the location, magnitude, and decay of the vortices being studied.

OVERFLOW was run on a Compaq Alpha 500 $\mathrm{MHz}$ machine for this study. The total run time for each case was approximately 40 hours. The parallel version of OVERFLOW, developed by Jespersen [11], was not used for this study because the source term model needed to be tested on a single processor first.

\section{Flat Plate Results}

The results of the source term model simulations were compared to experimental data and a simulation where a grid was generated for the VG. The experimental data was taken at four planes downstream of the VG using stereo digital particle image velocimetry (SDPIV) [12]. Figure 5 shows a schematic of the locations used for taking experimental data.

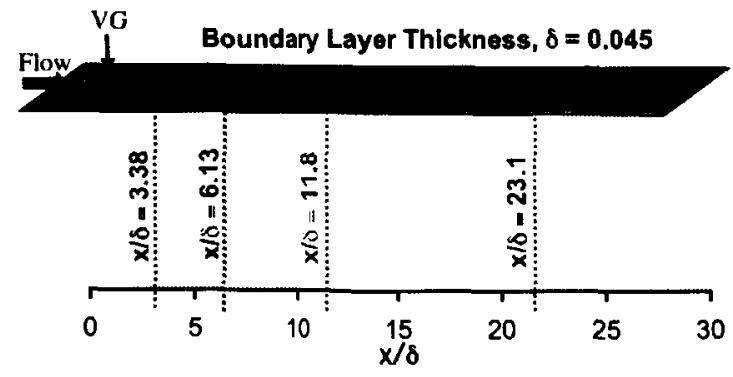

Figure 5. Schematic of location planes downstream of $V G$ for experimental data collection.

The source term model was calibrated before comparisons were made. To calibrate the model, Bender et al. [3] suggested that a calibration constant, $c$, be used to scale the side force in the source term model. In particular, Bender suggested plotting the effects of the normalized, cross-stream kinetic energy, $K$, downstream of the VG as the calibration constant is increased. As the calibration constant is increased, the cross-stream kinetic energy downstream of the source term should asymptote to a constant value. The source term model is correctly calibrated by using values of the calibration constant that occur in the asymptotic region of the crossstream kinetic energy plot. The normalized, crossstream kinetic energy is given by equation 2 .

$$
K=\frac{\int_{A} \rho\left(v^{2}+w^{2}\right) d A}{\int_{A} \rho u^{2} d A}
$$

In equation $2, \mathrm{u}, \mathrm{v}$, and $\mathrm{w}$ are the components of the local velocity vector, $\bar{U}, \rho$ is the density of the flow field, and $A$ is the area of the cross-section over which the cross-stream kinetic energy is integrated. Figure 6 shows the square root of the normalized, cross-stream kinetic energy, $\mathrm{K}^{1 / 2}$, versus the calibration constant, $\mathrm{c}$, at various grid cell widths or number of rows that span the chord of the VG being modeled. The number of rows needed to span the chord of the VG being modeled will vary depending on the coarseness of the grid. For the best results, the cross-stream-stream kinetic energy of the model and the gridded VG should be compared. In particular, once a value of a calibration constant causes the curve of figure 6 to asymptote, the user may then modify the number of rows of cells spanning the chord of the VG being modeled until the asymptotic region matches the cross-stream kinetic energy of the gridded VG. In figure 6, the cases with 3 grid cells wide asymptotes to the cross-stream kinetic energy of the gridded VG. The case with 3 grid cells wide and a calibration constant, $c=10$ will be used for comparison with experimental data and CFD data of the gridded VG.

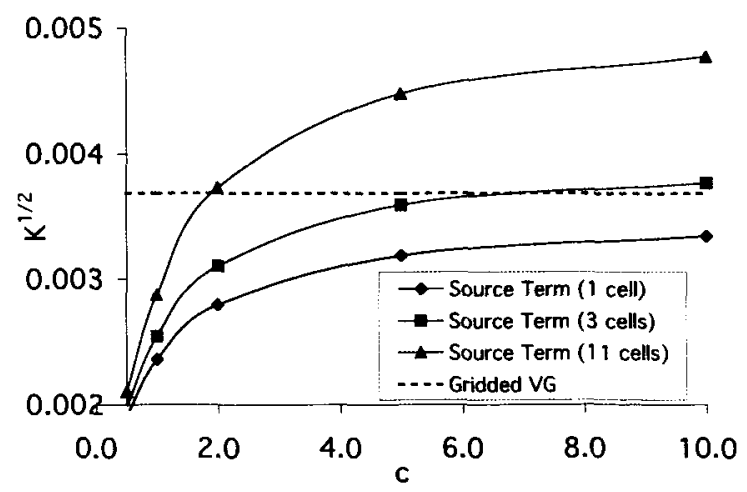

Figure 6. Square root of cross-stream kinetic energy $v s$, calibration constant, $c$. 
The circulation, $\Gamma$, which characterizes the decay of the vortex strength, and the stream-wise vorticity, $\omega$, were computed for the source term model by equations 3 and 4 respectively.

$$
\begin{aligned}
& \Gamma=\int_{A}\left(\frac{\partial v}{\partial z}-\frac{\partial w}{\partial y}\right) d A \\
& \omega=\frac{\partial v}{\partial z}-\frac{\partial w}{\partial y}
\end{aligned}
$$

In equations 3 and $4, v$ and $w$ are the components of the local velocity vector, $\bar{U}, \mathrm{y}$ and $\mathrm{z}$ are the crossstream directions perpendicular to each other and to the axial direction, $x$, and $A$ is the area of the crosssection over which the circulation is integrated.

Figure 7 shows a comparison of the positive circulation between the source term model, the experiment, and the gridded VG. The axial location, $\mathrm{x}$, is nondimensionalized by the boundary layer thickness, $\delta=0.045 \mathrm{~m}$. The source term model compares very well with the gridded VG. Although, both the source term model and the gridded VG do not compare well with the experiment upstream, they compare well with each other. This comparison helps to validate the use of the source term model as an alternative to generating a grid for the VG.

The peak stream-wise vorticity, $\omega_{\max }$, and its location are shown in figure 8. The plots are also nondimensionalized by the boundary layer thickness, $\delta=0.045 \mathrm{~m}$. The source term predicts the vortex trajectory very well; details of peak vorticity are not resolved near the VG, but far away the effects are the same.

Figures 9 and 10 show the stream-wise velocity, $u$, and the stream-wise vorticity, $\omega$, contours at various locations downstream from the VG. The source term model predicts the size and shape of both contours except at the stream-wise velocity contour immediately downstream of the VG at $\mathrm{x} / \delta=3.38$. The shape at this location is not as well defined as both the experiment and the gridded VG.

The flow residual history of the case with 3 grid cells wide and $c=10$ is shown in figure 11. The flow residuals of the other cases are similar. The residual history is used to track the convergence of the CFD solution. A residual drop of several orders of magnitude is expected from the $1 / 4$ grid resolved solution to the full grid resolved solution of a converged solution. The solution has dropped four orders of magnitude for the block grid that captures the vortex and three orders of magnitude for the flat plate grid.

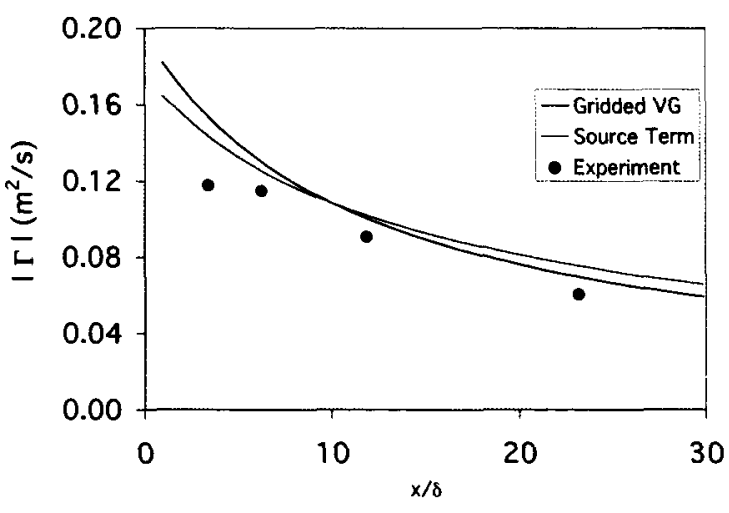

Figure 7. Circulation vs. axial location.
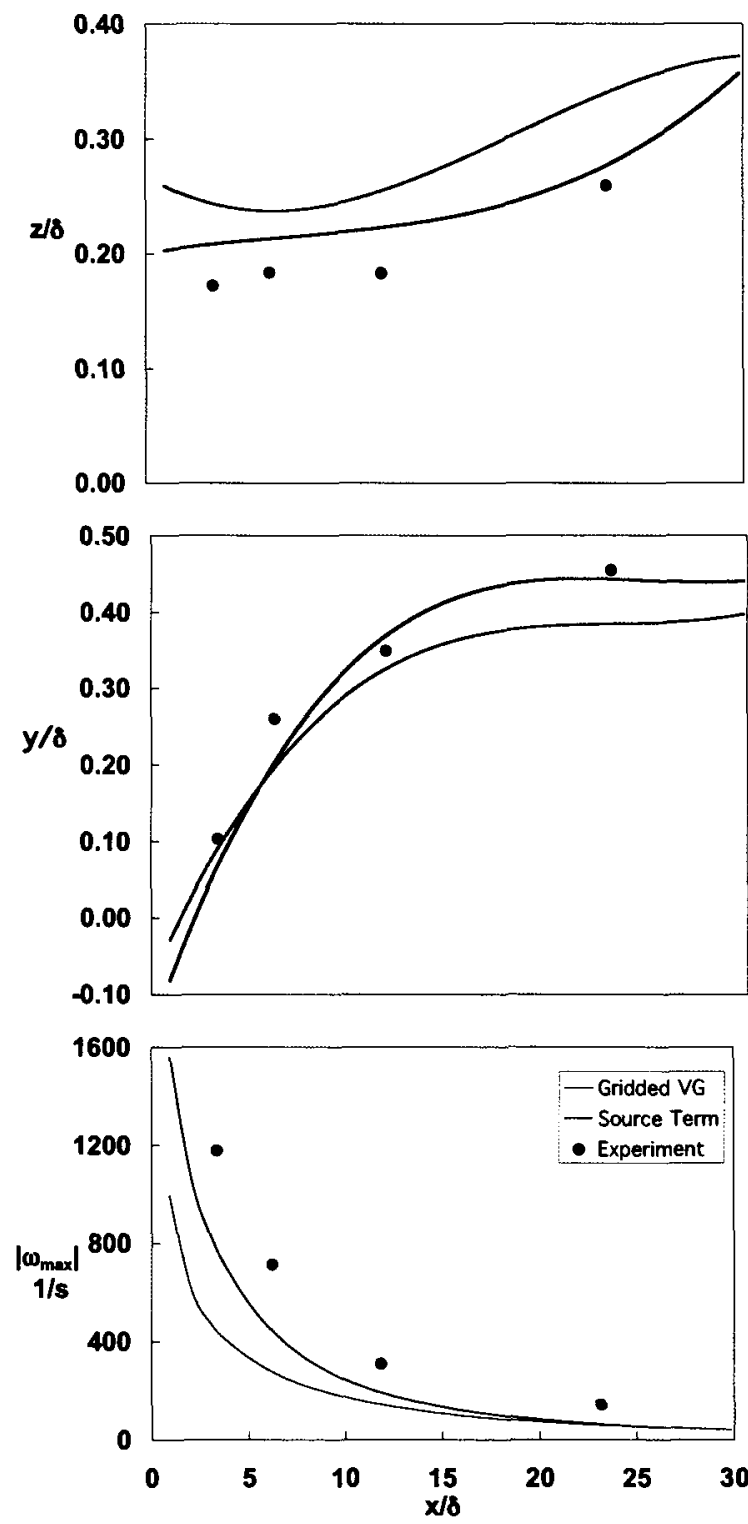

Figure 8. Location of Peak Stream-wise vorticity. 


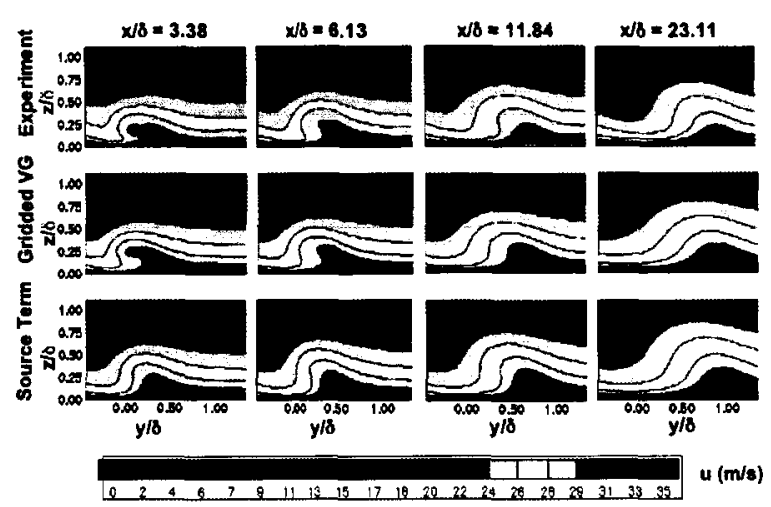

Figure 9. Stream-wise velocity contours downstream of VG at four axial locations.

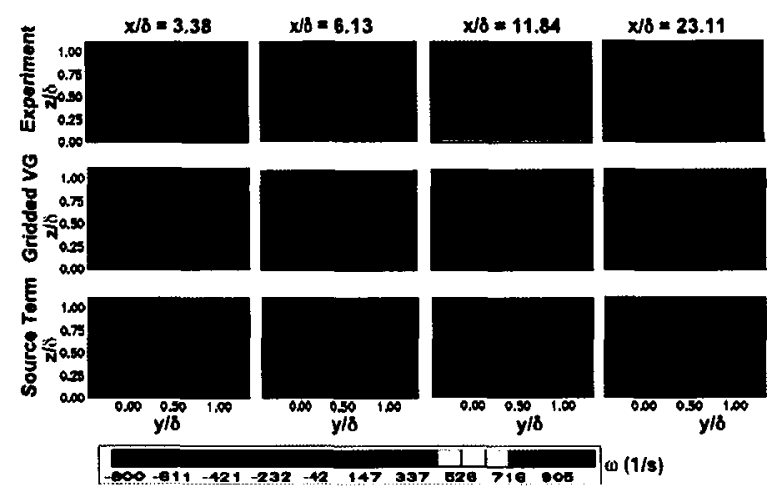

Figure 10. Stream-wise vorticity contours downstream of VG at four axial locations.

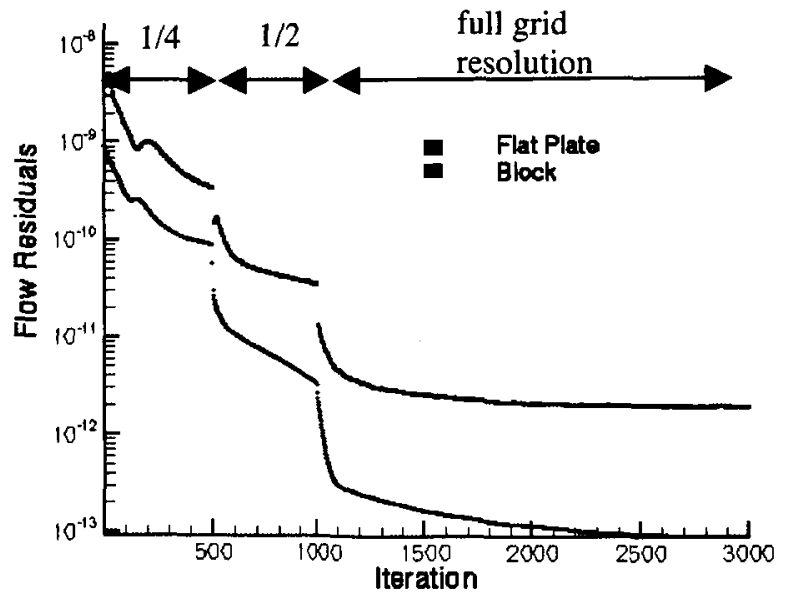

Figure 11. CFD flow residual history.

\section{M2129 Inlet Study}

The source term model was also tested by running simulations on an S-duct, the M2129 inlet. A source term was used to model 22 co-rotating low profile VGs. The grid for the M2129 inlet used in this study is shown in figure 12 and comprises a single grid of 252,154 grid cells.

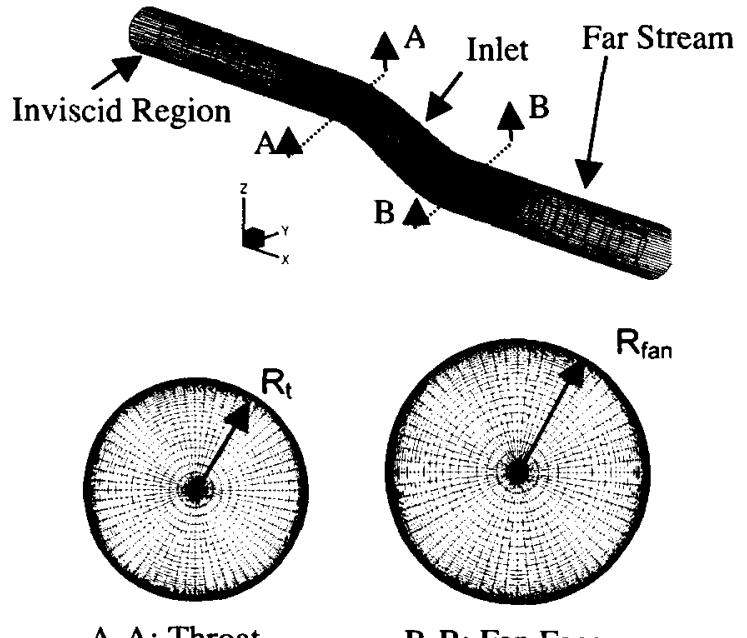

A-A: Throat

B-B: Fan Face

Figure 12. Computational domain for M2129 inlet study.

The inviscid region of the computational domain is to insure uniform flow at the inlet of the S-duct, which has a radius, $R_{t}=2.54$ ". The far stream region of the computational domain is to ensure there is enough computational space for the solution to be extrapolated. The radius of the fan face is $\mathrm{R}_{\text {fan }}=3.00$ ".

A schematic of one of the $22 \mathrm{VGs}$ modeled is shown in figure 13 , which has a length to height ratio of 4 , a planform area of $0.1265 \mathrm{in}^{2}$, and a $16^{\circ}$ angle of attack relative to the local flow.
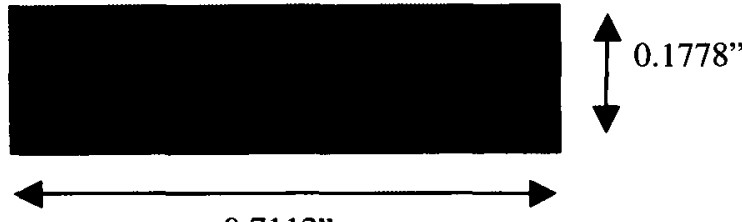

$0.7112 "$

Figure 13. Schematic of one of 22 co-rotating VGs modeled.

Figure 14 shows a schematic of the location and orientation of the VGs in the M2129 inlet.

A total of 10 cases were run at Mach numbers of $\mathrm{M}=0.4,0.5,0.6,0.7$, and 0.8 with and without the source term model and a Reynolds number of 16 million per foot, which was the test condition. The cases without the source term model provided a baseline set of data for the inlet without VGs and the cases with the source term model provided data for the simulation of 22 co-rotating VGs in the inlet. 


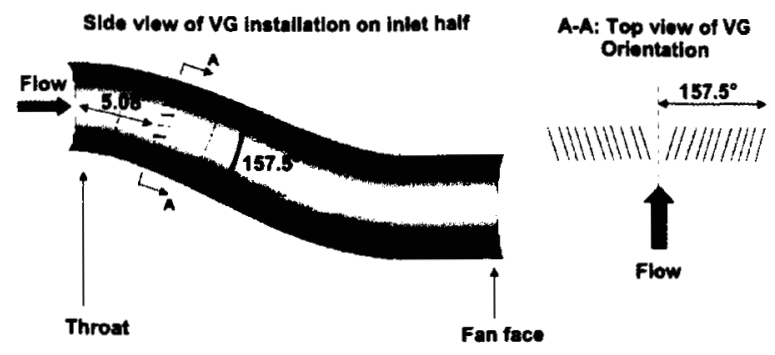

Figure 14. Schematic of VG location and orientation in the M2129 inlet.

The low-Mach preconditioning and multi-grid acceleration options used in the flat plate study were used again to improve the convergence of the steadystate solution. Also, all simulations were run using the two-equation $(\kappa-\omega)$ SST turbulence model of Menter for the reasons discussed in the flat plate study.

OVERFLOW was run on a Compaq Alpha 500 $\mathrm{MHz}$ machine for this study. The total run time for each case was approximately 4.7 hours.

\section{M2129 Inlet Results}

The results of the source term model simulations were compared to experimental data on the M2129 inlet. The experimental pressure data was taken at the engine fan face using a 72-probe rake. All CFD data that was used for comparison with experimental data was interpolated using the 72-probe locations on the engine fan face of the inlet. Figure 15 shows a schematic of the 72-probe locations on the engine fan face.

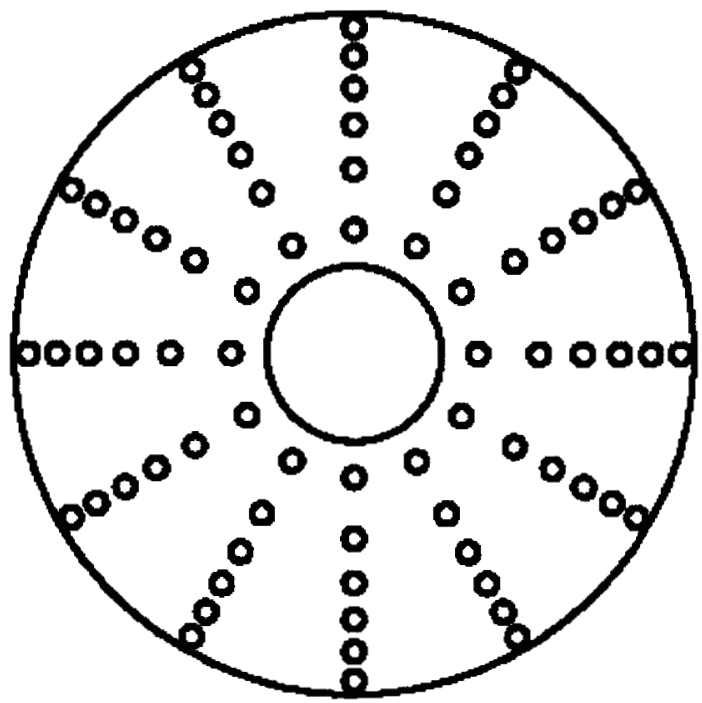

Figure 15. Schematic of 72-probe rake locations.
Figures 16 and 17 show the CFD fan face total pressure contours for $\mathrm{M}=\mathbf{0 . 4}$ and $\mathrm{M}=0.8$, respectively.

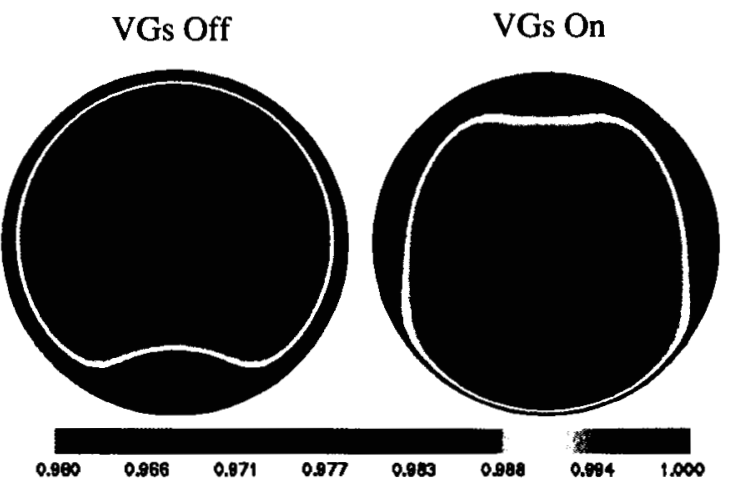

Figure 16. CFD fan face total pressure contours, $p_{0} / p_{0, \infty}$ for $M=0.4$ with and without VGs.

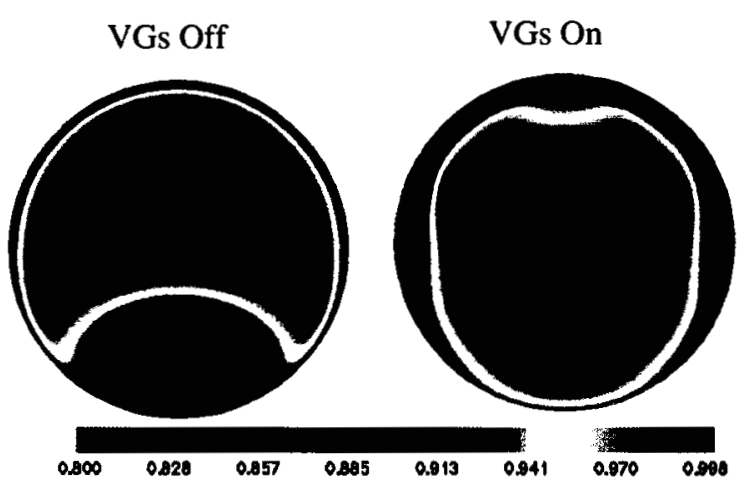

Figure 17. CFD fan face total pressure contours, $p_{0} / p_{0, \infty}$ for $M=0.8$ with and without VGs.

The application of the VGs redistributes the flow more evenly over the fan face, reducing the low pressure region at the bottom of the fan face.

Figures 18 and 19 show the CFD fan face total pressure contours at various axial locations along the inlet and streamlines of the flow for $M=0.4$ and 0.8 , respectively. The M2129 inlet has flow separation without the VGs, which is evident by the "lift-off" of the streamlines in figures 18 and 19. The flow separation is the reason for uneven total pressure distribution over the fan face shown in figures 16 and 17 , which leads to a high fan face distortion and low fan face pressure recovery. The application of the VGs keeps the flow attached, which is evident by the streamlines in figures 18 and 19. The flow is more evenly distributed at the fan face because of the flow attachment as shown in figures 16 and 17 , which results in a lower fan face distortion and higher fan face pressure recovery.

American Institute of Aeronautics and Astronautics 

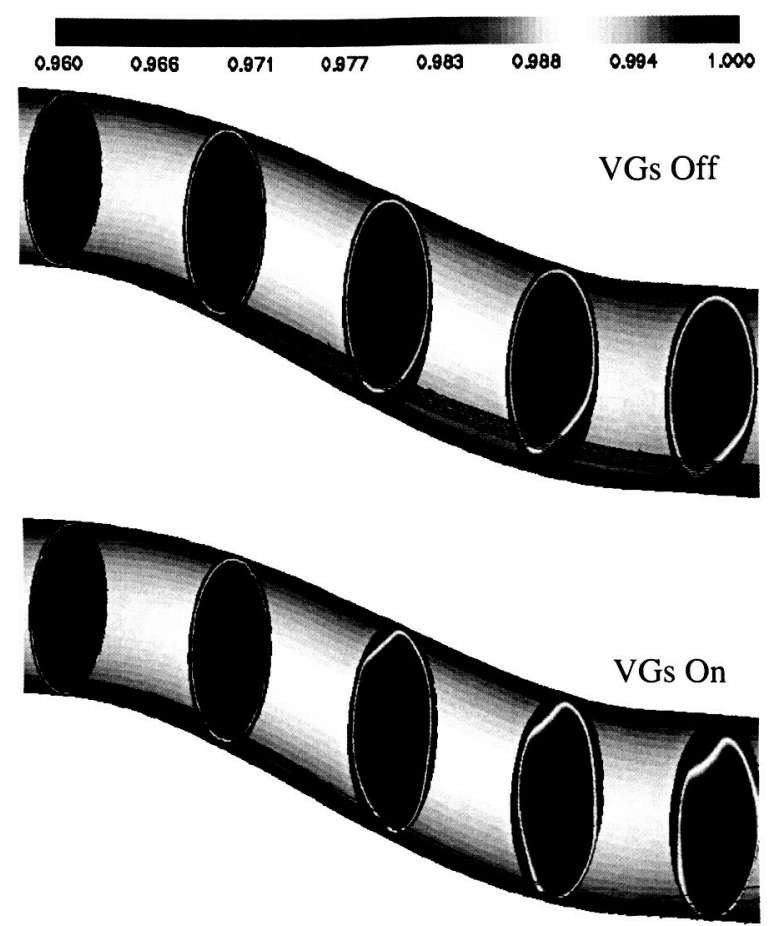

Figure 18. CFD fan face total pressure contours, $p_{0} / p_{0, \infty}$ at various axial locations along inlet and flow streamlines for $M=0.4$ with and without VGs.
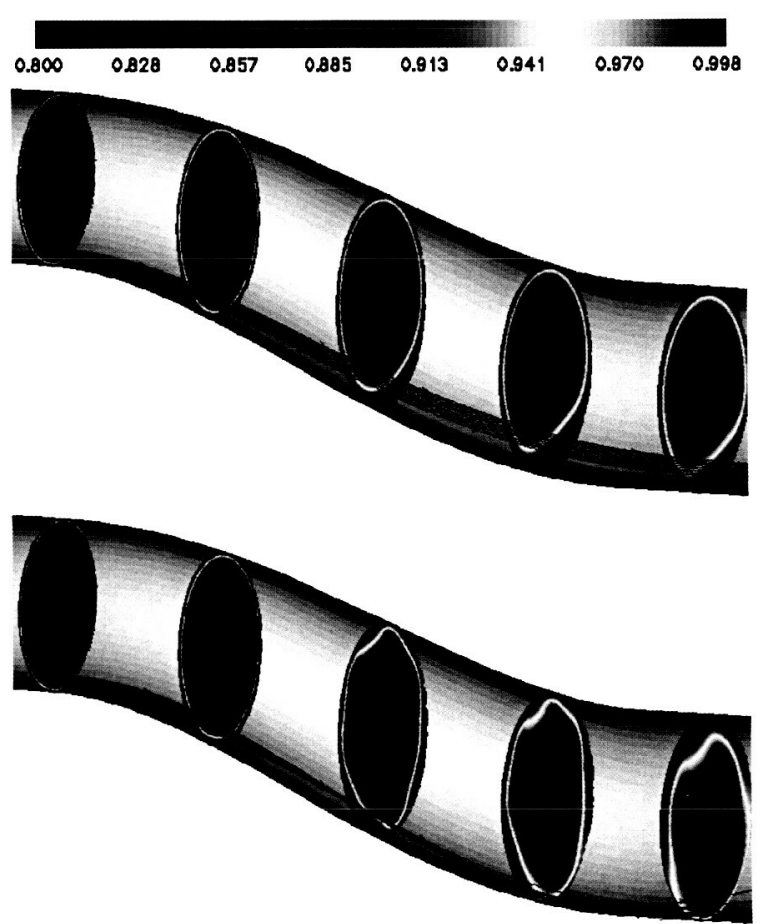

Figure 19. CFD fan face total pressure contours, $p_{0} / p_{0, \infty}$ at various axial locations along inlet and flow streamlines for $M=0.8$ with and without VGs.
Figure 20 shows the engine fan face distortion, $\mathrm{DC}_{60}$ descriptor, versus the inlet Mach number for the experiment and CFD simulations with and without VGs.

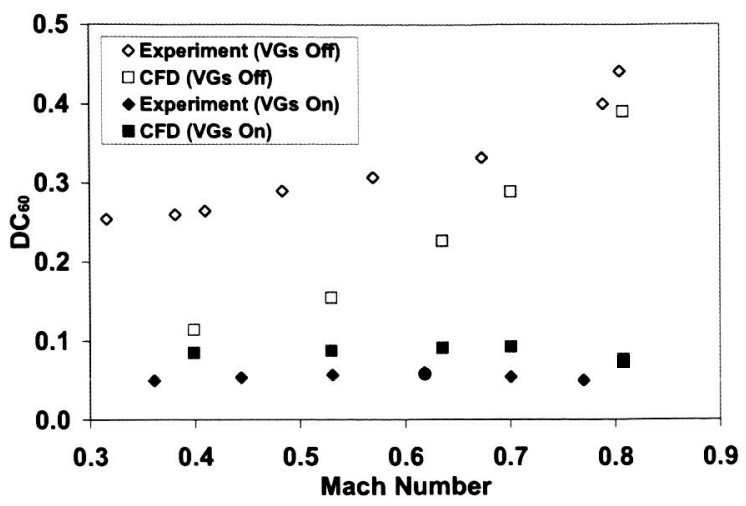

Figure 20. Engine fan face distortion, $D C_{60}$, versus Mach number with and without VGs.

In figure 20, the application of VGs causes the fan face distortion to decrease at all Mach numbers. The source term model (CFD with VGs on) did a very good job predicting the fan face distortion when compared to experimental results. However, the CFD simulations without VGs did a poor job at low Mach numbers predicting the fan face distortion when compared to experiment. One possible reason for the discrepancy between CFD and experiment could be attributed to the turbulence model used in OVERFLOW. In particular, at low Mach numbers the turbulence model may be under predicting the separation in the inlet. Another CFD code, PAB3D [13] with a two-equation $(\kappa-\varepsilon)$ turbulence model, was run using the same computational domain to determine if turbulence modeling was a factor in the discrepancy between CFD and experiment. The twoequation $(\kappa-\varepsilon)$ model in PAB3D is known for accurate turbulence modeling of internally separated flows.

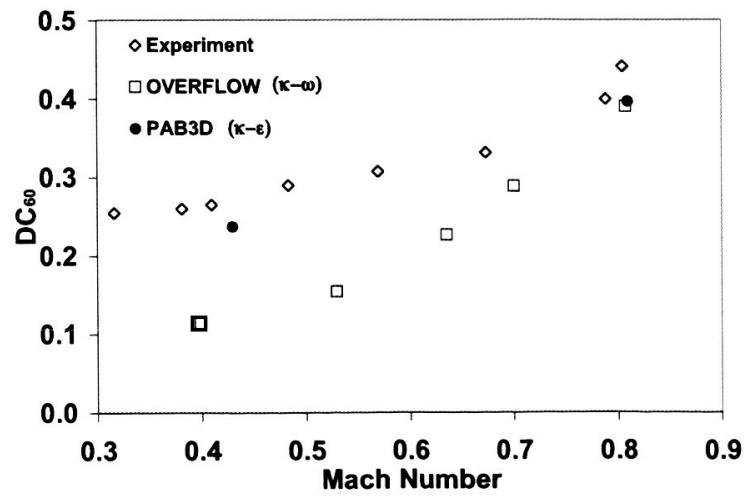

Figure 21. Engine fan face distortion, $D C_{60}$, versus Mach number without VGs. 
Figure 21 shows the engine fan face distortion versus Mach number for the experiment, OVERFLOW, and PAB3D without VGs. The results of figure 21 indicate that turbulence modeling is the main factor in the discrepancy between OVERFLOW and the experimental results with no VGs. The cases with VGs eliminate the separation, which is why OVERFLOW does a better job predicting the fan face distortion at all Mach numbers.

Figure 22 shows the fan face total pressure recovery, PFAVE, versus Mach number for the experiment and CFD simulations with and without VGs.

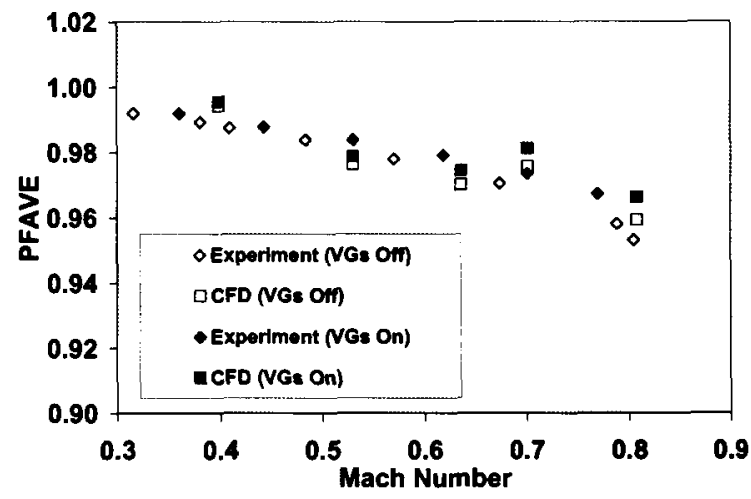

Figure 22. Fan face total pressure recovery, PFAVE, versus Mach number with and without VGs.

In figure 22, the application of the VGs causes a higher total pressure recovery, which is evident from both the experiment and the CFD results. The CFD simulations did a good job when compared with the experiment in predicting the fan face pressure recovery for all Mach numbers with and without VGs.

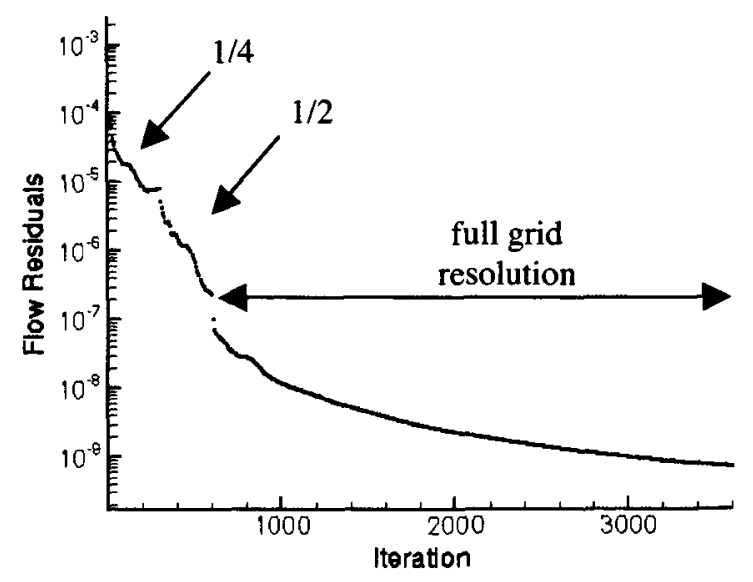

Figure 23. CFD flow residual history. cases are similar. As mentioned previously, the residual history is used to track the convergence of the CFD solution. A residual drop of several orders of magnitude is expected from the $1 / 4$ grid resolved solution to the full grid resolved solution of a converged solution. As shown in figure 23 , the solution has dropped five orders of magnitude, indicating a converged solution.

\section{BLI Results}

A boundary layer ingestion inlet (BLI) is currently being investigated at NASA Langley Research Center [6]. The BLI inlet has a very nonuniform pressure distribution at the engine fan face due to ingestion of the boundary layer, which leads to a high engine fan face distortion. Investigators at Langley are researching the placement of VGs and micro jets in an effort to more evenly distribute the flow at the engine fan face and reduce distortion.

The following results were obtained by Brian Allan of NASA Langley Research Center. The results are presented to illustrate the use of the source term model as a design tool. In particular, the source term model was utilized to investigate the placement of rows of VG jets in the BLI inlet in order to distribute the flow more evenly over the fan face and reduce the fan face distortion. Although the source term model currently only models the effects of vortex generator vanes, it will guide the researchers in placing the jets in the inlet. The final results will aid investigators in selecting configurations of jets to be placed in a BLI inlet model, which will be tested in the wind tunnel. Figures 24 to 28 show the CFD total pressure contours of the CFD simulations with and without the application of various VG patterns.

The source term model allows the investigator to quickly look at possible VG locations without the expense of generating a grid for the VGs. As a result, the computational domain is reduced from 14 million grid cells ( 7 jets gridded) to only 1.1 million grid cells. In addition, solutions are obtained in about 1 hour using 6 Intel $2.8 \mathrm{GHz}$ Pentium 4 processors versus 24 hours using 24 Intel $2.8 \mathrm{GHz}$ Pentium 4 processors. All simulations were run at a Mach number, $M=0.83$ and a Reynolds number of 13.9 million.

The flow residual history of the Mach $=0.4$ case is shown in figure 23. The residuals of the other 


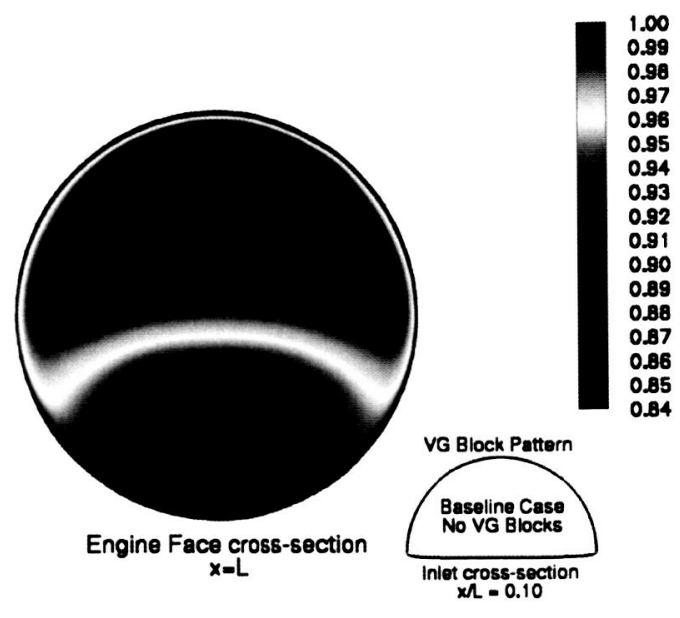

Figure 24. CFD fan face total pressure contour, $p_{0} / p_{0, x}$ without VGs.

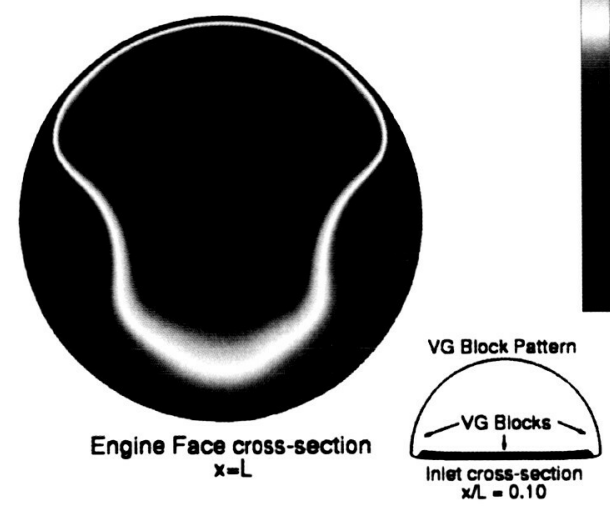

Figure 25. CFD fan face total pressure contour, $p_{0} / p_{0, \infty}$ with row of VGs on the bottom.

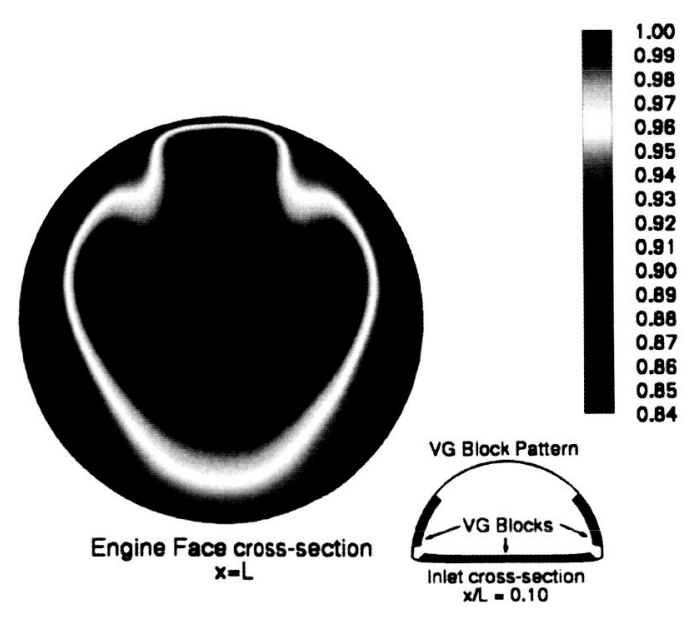

Figure 26. CFD fan face total pressure contour, $p_{0} / p_{0, \infty}$ with row of VGs on the bottom and half way up the sides.

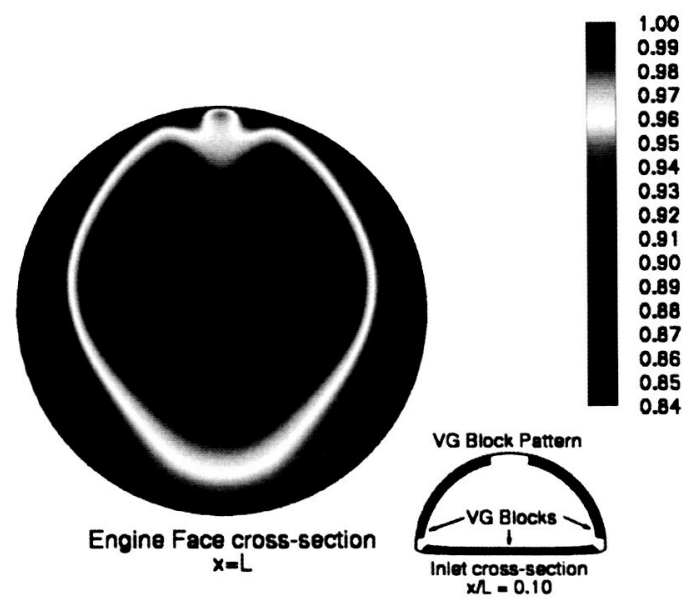

Figure 27. CFD fan face total pressure contour, $p_{0} / p_{0, \infty}$ with row of VGs on the bottom and sides.

\section{Conclusions}

In general, the source term model predicted the size, shape, location, and decay of the streamwise vorticity of the VG on a flat plate and is a viable alternative to a fully gridded VG solution. The fully gridded solution used for comparison in the flat plate study comprised a total of $6,405,100$ grid cells. The source term model realized a 70 percent reduction in grid cells without any significant drop in predicting the flow field.

The source term model can predict the effects of VGs in an S-duct, such as the M2129 inlet. In particular, the model did a very good job predicting the reduction in fan face distortion and improved pressure recovery caused by the application of VGs without having to generate a grid for the VGs.

The BLI inlet results serve to demonstrate the source term model as a design tool. The use of the source term model allows the investigator to research the locations of VGs in $1 / 24$ of the time it takes to obtain a solution with a fully gridded VG. In addition, the time to generate grids for various VG configurations is eliminated.

The source term model is a viable tool for investigating $\mathrm{VG}$ installations in an inlet or on a surface. The model significantly reduces the turnaround time from conception to solution by reducing grid generation and computational time. 


\section{Acknowledgements}

The National Aeronautics and Space Administration Ultra Efficient Engine Technology Project sponsored this work. The author would like to acknowledge Mark Chaffin and Pieter Buning for all of their help in implementing the source term model into OVERFLOW. Brian Allan of NASA Langley and Bernie Anderson of NASA Glenn are acknowledged for their help in providing data for comparisons with the model. Also Susan Gorton is acknowledged for her help and guidance in this effort. The Configuration Aerodynamics Branch of the National Aeronautics and Space Administration Langley Research Center is acknowledged for their support on this project.

\section{References}

1. Hamstra, J. W., Miller, D. N., Truax, P. P., Anderson, B. H., and Wendt, B. J., Acitve Inlet Flow Control Technology Demonstration. ICAS-2000-6.11.2, 2000.

2. Allan, B.G., Yao, C.S., and Lin, J.C, Numerical Simulations of Vortex Generator Vanes and Jets on a Flat Plate, AIAA Paper 2002-3160, June 2002.

3. Bender, E. E., Anderson, B. H., and Yagle, P. J., Vortex Generator Modeling for Navier-Stokes Codes. FEDSSM99-6919, 1999.

4. Buning, P.G., Jespersen, D. C., Pulliam, T. H., Klopfer, W.M., Chan, W. M., Slotnick, J. P., Krist, S. E., and Renze, K. J., OVERFLOW User's Manual Version $1.8 s$, NASA Langley Research Center, Hampton, VA, November 2000.

5. Anderson, B. H. and Gibb, J, Vortex Generator Installation Studies on Steady State and Dynamic Inlet Distortion, NASA TM 107220, 1996.

6. Berrier, B. L. and Allan, B. G., Experimental and Computational Evaluation of FlushMounted, S-Duct Inlets, AIAA Paper 2004-0764, 2004.

7. Pulliam, T. H. and Chaussee, D. S., A Diagonal Form of an Implicit Approximate-Factorization Algorithm, Journal of Computational Physics, Vol. 39, No.2, pp. 347-363, 1981.

8. Steger, J. L., Dougherty, F. C., and Benek, J. A., A Chimera Grid Scheme, in Advances in Grid Generation, K. N. Ghia and U. Ghia, eds., vol. 5 of FED ASME, New York, NY, 1983.

9. Menter, F., Improved Two-Equation turbulence Models for Aerodynamic Flows, NASA TM 103975, 1992.
10. Spalart, P. and Allmara, S., A One-Equation turbulence Model for Aerodynamic flows, La Recherche Aerospatiale, No.1, pp. 5-21, 1994.

11. Jespersen, D. C., Parallelism and OVERFLOW, Tech. Report NAS-98-013, NASA Ames Research Center, Moffett Field, CA 23681-2199, October 1998.

12. Yao, C-S, Lin, J.C., and Allan, Brian A., FlowField Measurement of Device-Induced Embedded Streamwise Vortex on a Flat Plate, AIAA Paper 2002-3162, 2002.

13. Abdol-Hamid, Khaled S., Application of a Multiblock/Multizone Code (PAB3D) for ThreeDimensional Navier-Stokes Equations, AIAA Paper 91-2155, June 1991. 